\title{
Accuracy of the CT numbers of simulated lung nodules imaged with multi-detector CT scanners
}

\author{
Mitchell M. Goodsitt, ${ }^{\text {a) }}$ Heang-Ping Chan, Ted W. Way, Sandra C. Larson, \\ Emmanuel G. Christodoulou, and Jeomsoon Kim \\ Department of Radiology, University of Michigan, Ann Arbor, Michigan 48109
}

(Received 14 March 2006; revised 15 May 2006; accepted for publication 9 June 2006; published 27 July 2006)

A study was performed to determine the accuracies and reproducibilities of the CT numbers of simulated lung nodules imaged with multi-detector CT scanners. The nodules were simulated by spherical balls of three diameters $(4.8,9.5$, and $16 \mathrm{~mm})$ and two compositions $(50$ and $100 \mathrm{mg} / \mathrm{cc}$ $\mathrm{CaCO}_{3}$ in water-equivalent plastic). All were scanned in a liquid-water-filled container at the center of a water-equivalent-plastic phantom and in air cavities within the same phantom using GE multidetector CT scanners. The nodules were also scanned within simulated lung regions in an anthropomorphic thorax section phantom that was bolused on both sides with water-equivalent slabs. Results were compared for three scanning protocols - the protocol for the National Lung Screening Trial (NLST), the protocol for the Lung Tissue Research Consortium (LTRC) study, and a high resolution (small pitch, thin slice and small scan interval) higher dose "gold standard" protocol. Scans were repeated three times with each protocol to assess reproducibility. The CT numbers of the nodules in water were found to be nearly independent of nodule size. However, the presence and the size of an air cavity surrounding a nodule had a significant effect (e.g., the CT number of a $50 \mathrm{mg} / \mathrm{cc}$ nodule was $64 \mathrm{HU}$ in water, $37 \mathrm{HU}$ in a $1.8 \mathrm{~cm}$ diameter air cavity, and $19 \mathrm{HU}$ in a $4.4 \mathrm{~cm}$ diameter air cavity). This variability of CT number with size of air cavity may affect the results of the LTRC study in which patients are scanned at both full inspiration and full expiration. The CT numbers of the 9.5 and $16 \mathrm{~mm}$ diameter nodules within the anthropomorphic phantom were highly reproducible (average standard deviations of $2 \mathrm{HU}$ or less) for all protocols. On the other hand, both accuracy and reproducibility were significantly degraded for the $4.8 \mathrm{~mm}$ diameter nodules, especially for the NLST $(2.5 \mathrm{~mm}$ thickness, $2 \mathrm{~mm}$ slice interval) technique. Use of thinner slice $(1.25 \mathrm{~mm})$ and slice interval $(1.25 \mathrm{~mm})$ scans that can be reconstructed retrospectively from the multi-detector helical CT projection data of the standard NLST protocol yield CT numbers for the $4.8 \mathrm{~mm}$ diameter nodules that are more accurate and reproducible than those of the standard NLST technique. In general, the CT numbers of the nodules were found to be lower at positions near the centers of the lungs and near the spine, which is probably due to increased beam hardening in those regions. Also, larger nodules were found to have higher CT numbers than smaller nodules, consistent with results obtained on early single slice GE CT scanners. Until manufacturers develop quantitative CT scanners with improved x-ray beam hardening and scatter corrections, it is recommended that reference phantoms be employed to more accurately assess the calcium contents of patient lung nodules in screening and tissue characterization studies and in eventual computer-aided detection and diagnosis applications. (C) 2006 American Association of Physicists in Medicine. [DOI: $10.1118 / 1.2219332]$

Key words: computed tomography, lung nodule, phantoms

\section{INTRODUCTION}

The Computer Aided Diagnosis (CAD) Research Laboratory at the University of Michigan is developing algorithms to detect and characterize lung nodules in CT images. One of the distinguishing features of many benign pulmonary nodules is the presence of a significant amount of calcifications with central, diffuse, laminated, or popcornlike patterns. ${ }^{1}$ Calcium salts are accumulated by dead or dying benign nodules because as the cells die, the proteins are denatured, exposing "groups capable of binding phosphates which in turn serve as sites for the deposition of calcium in the form of phosphates, carbonates, or oxalates." ${ }^{2}$ Since calcium absorbs $\mathrm{x}$ rays more than most other tissue components, it can often be readily detected in CT images. The pixel values (CT numbers) of a CT image are directly related to the relative x-ray attenuation of the tissues. Ideally, the CT number of a tissue should depend only upon the composition of the tissue. Unfortunately, this is not the case. Many other factors affect the CT numbers including $\mathrm{x}$-ray beam hardening, $\mathrm{x}$-ray scatter, partial volume, and reconstruction kernel effects. These factors cause errors in the CT numbers, which can reduce the conspicuity of calcifications in pulmonary nodules.

One way to counter these effects is to relate the CT numbers in a patient scan to those in an anthropomorphic phantom. Zerhouni et al. ${ }^{3,4}$ developed such a phantom for pulmo- 
nary nodules and employed it in studies with considerable success. The reference phantom became a commercial product marketed by Computerized Imaging Reference Systems, Inc. (CIRS) of Norfolk, VA. It consists of three x-ray tissueequivalent transaxial sections that simulate the anatomy of the thorax in the upper, middle, and lower levels. These sections simulate small patients. The reference phantom includes sets of fat-equivalent rings that can be added to the outside of the sections so as to simulate medium- and largesize patients. There are also three different size inserts for simulating the liver, spleen, and diaphragm. In addition, there is a set of 15 reference nodules. These reference nodules are cylinders or rods of various diameters that are made of epoxy resins. A certain amount of calcium carbonate $\left(\mathrm{CaCO}_{3}\right)$ was mixed in the resin to produce a desired threshold CT number for distinguishing benign from malignant nodules. The amount of $\mathrm{CaCO}_{3}$ was experimentally determined on a Pfizer/AS\&E $500 \mathrm{CT}$ scanner. In the initial research phantom, ${ }^{3}$ the desired threshold was set at $164 \mathrm{HU}$ for the $1 \mathrm{~cm}$ diameter rod. This value was determined from earlier patient studies. ${ }^{5}$ Later, to add a margin of safety (to reduce the number of false benign diagnoses and account for overestimates of the CT numbers of small nodules by the Pfizer/AS\&E scanner and CT number variability for other scanners), the composition was adjusted to produce a representative CT number (=average of ten highest pixel values) of $264 \mathrm{HU}$ for the $1 \mathrm{~cm}$ reference nodules when scanned in the center of the lung in the thorax section phantom using the Pfizer/AS\&E scanner. ${ }^{4}$ The CT numbers of the reference nodules did vary considerably with the size of the nodule and the type of scanner. For example, the CT numbers of the 6,8 , 10 , and $20 \mathrm{~mm}$ diameter cylindrical reference nodules measured on a Pfizer/AS\&E 500 scanner were 310, 284, 264, and $190 \mathrm{HU}$, respectively, and the corresponding values on a GE 9800 scanner were 74, 83, 97, and $145 \mathrm{HU}^{4}$ The threshold that was decided upon for the commercial product was a representative CT number of $185 \mathrm{HU}^{6}{ }^{6}$ The amount of $\mathrm{CaCO}_{3}$ was reduced from that in the $264 \mathrm{HU}$ reference nodules "in order to increase the percentage of calcified nodules diagnosed as benign with the reference phantom."

The reference phantom CT method may be summarized as follows. First, the patient is scanned. Next, the patient's images are examined for suspicious nodules. When a slice containing a suspicious nodule is found, a reference phantom section that best matches this slice (including appropriate fat rings and liver, spleen, and diaphragm inserts where applicable) is selected. Next, a reference nodule of about the same size as the suspicious patient nodule is positioned at about the same location in the lung region of the phantom. Finally, the phantom is scanned using the same $\mathrm{x}$-ray technique factors as were used for the patient.

The criteria employed for classifying patient nodules as probably benign included (1) having smooth or lobulated borders in the CT image, (2) having CT numbers greater than the CT number of the corresponding scanned reference phantom nodule with the further requirement that these high CT numbers be in a benign pattern (i.e., central, laminated or diffuse), and if the high pixel values were centrally located, they had to represent at least $10 \%$ of the cross-sectional area, and (3) the benign pattern had to be present in at least two slices through the nodule., ${ }^{4,6}$

Researchers have had mixed results with the commercial phantom. Khan et al. ${ }^{7}$ compared the accuracy of the classification of calcified and noncalcified solitary pulmonary nodules obtained with standard CT (10 mm slice thickness), thin-section CT (1.5 mm slice thickness), and reference phantom CT. The latter utilized the thin-section CT images of the patient and corresponding thin-section CT images of an appropriate reference thorax section phantom. That phantom contained a cylindrical reference nodule, the size and lung location of which were determined from the CT images of the patient. Khan et al. found that the reference phantom technique of Zerhouni et al. improved sensitivity by $22 \%$ compared with thin-section CT, which was the next best method. On the other hand, in a different study, Swensen et al., ${ }^{6}$ found that 10 of 85 cases that were diagnosed as benign by comparison with the reference phantom were later shown to be malignant. This represented a much higher misdiagnosis rate than in other studies, and Swensen et al. cautioned that even if the reference phantom indicated a high probability of benignity, those nodules should be considered indeterminate and should be closely followed up.

Today, thoracic scans are conventionally performed using multi-detector CT in helical mode, technologies that did not exist when the reference phantom was developed. Also, the new scanners employ high-frequency generators with $\mathrm{kVp}$ and $\mathrm{mA}$ feedback loops that make the exposures and therefore the reconstructions more reproducible. However, to our knowledge, CT scanner manufacturers still have not addressed the CT number inaccuracy issue. For our CAD application, our long-term goal is to compensate for variations in the CT numbers of the nodules with position in the lung field and with the size of the nodules by employing a modified reference phantom to determine CT number versus calcium concentration calibration curves throughout the lung fields. The curves will be used to convert the CT numbers of the voxels in the patient lung nodules to calcium concentrations, the values and distributions of which in turn will be employed as CAD features. The commercial reference phantom was modified for our studies by increasing the phantom thickness to account for the longer scan lengths associated with helical scanning with pitches greater than 1. Also, spherical rather than cylindrical reference nodules were employed in order to better simulate the shape of patient nodules and the associated partial volume effects on the estimation of the CT numbers.

It should be noted that the spherical reference nodules that were employed in our studies were uniform mixtures of $\mathrm{CaCO}_{3}$ in water-equivalent plastic. Therefore, they simulated "solid" solitary pulmonary nodules and are representative of only one of the three classes of nodules that are detected at screening. These classes include ground glass opacity, focal ground glass opacity with a solid central component, and solid nodules. ${ }^{8}$ Nevertheless, the CT-number-to$\mathrm{CaCO}_{3}$-concentration conversion relations that are derived 


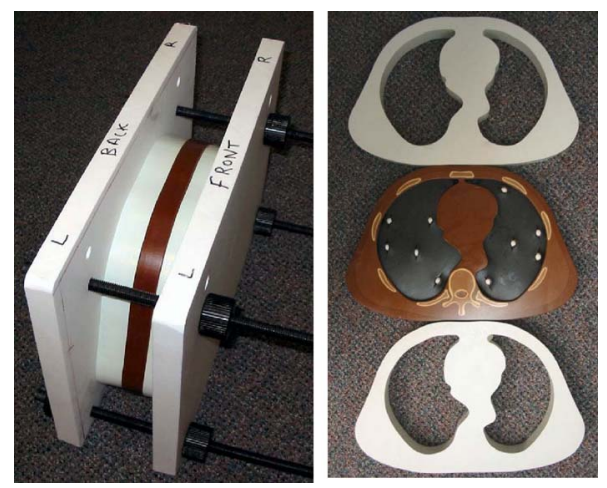

FIG. 1. Left: Setup for CT scanning showing thorax section sandwiched between two water-equivalent bolus sections. The sections are tightly squeezed together using the outer plastic vice. Right: Front views of the two bolus sections and thorax section containing the $9.5 \mathrm{~mm}$ diameter spherical reference nodules with calcium carbonate concentrations of $50 \mathrm{mg} / \mathrm{cc}$ in the lung simulating foam on one side and $100 \mathrm{mg} / \mathrm{cc}$ on the other side.

with the reference nodules can be utilized to characterize entire nodules as well as regions-of-interest within the nodules in terms of calcium content. The calcium content should be less susceptible to factors that cause CT number variability such as differences in patient body size, nodule position within the lung, CT scanning parameters, CT scanner x-ray beam filtration, etc.

The purpose of the present study was to (1) develop the modified reference thorax phantom and nodules, (2) determine the uniformity of the CT numbers of the reference nodule samples and the dependence of the CT numbers on the sizes and calcium concentrations of the nodules, (3) determine the effect of the lung/air cavity on the measured CT numbers of the nodules, and (4) determine the reproducibility and dependence of the measured CT numbers of the reference nodules on their sizes, calcium concentrations, and positions within the thorax phantom for several multidetector helical CT scanning protocols.

\section{METHODS AND MATERIALS}

\section{A. Phantoms}

To make the commercial thorax phantoms, which are only $2.3 \mathrm{~cm}$ thick, more suitable for multi-detector helical CT scanning, we increased the thickness by adding bolus slabs to each side. We contracted CIRS, Inc. to build bolus sections in exactly the same shapes as the thorax sections. Due to cost considerations, we had the bolus sections made of water-equivalent plastic rather than anthropomorphic plastics simulating bone, muscle, and fat as in the thorax sections. Each bolus section is $2.9 \mathrm{~cm}$ thick, and the thorax section is sandwiched between 2 bolus sections to obtain a total thickness of $8.1 \mathrm{~cm}$. An example of a thorax section and two bolus sections is shown in Fig. 1.

In preliminary studies it was found that no matter how tightly we taped the bolus and thorax sections together, small gaps between the sections remained and produced streak artifacts in images created with helical scanning. To alleviate this problem, a plastic vice system was built to tightly squeeze the sections together. This system is shown in Fig. 1. Even with the vice some small gaps remained due to surface irregularities. These gaps were filled-in with petroleum jelly (a fatlike material), which was spread on the faces of the adjacent sections prior to squeezing them together.

\section{B. Reference nodules}

A wide variety of spherical reference nodules were manufactured for this study by CIRS, Inc. The nodules are composed of a water-equivalent plastic with known concentrations of calcium carbonate. The nodule sizes are 4.8, 9.5, and $16 \mathrm{~mm}$ in diameter. The smallest size is similar to the minimum size $(\geqslant 4 \mathrm{~mm})$ of a noncalcified nodule that "was considered a positive screen, i.e. suspicious for lung cancer" in the National Cancer Institute's Lung Screening Study. ${ }^{9}$ The $16 \mathrm{~mm}$ diameter nodules are in the largest size category of that study. The concentrations of $\mathrm{CaCO}_{3}$ are 50 and $100 \mathrm{mg} / \mathrm{cc}$. The $100 \mathrm{mg} / \mathrm{cc}$ concentration was selected to achieve a CT number slightly greater than the $97 \mathrm{HU}$ value for the reference nodule that was measured experimentally by Zerhouni et al. on a GE $9800 \mathrm{CT}$ scanner. ${ }^{4}$ The effective energy of this scanner is about $70 \mathrm{keV}$ at $120 \mathrm{kVp}$ and, using linear attenuation coefficients computed with XCOM [http:/ /physics.nist.gov/PhysRefData/Xcom/Text/XCOM.html] for a $100 \mathrm{mg} / \mathrm{cc} \mathrm{CaCO}_{3}$ in water mixture, the CT number is $118 \mathrm{HU}$. The $50 \mathrm{mg} / \mathrm{cc}$ concentration was selected because it is half of $100 \mathrm{mg} / \mathrm{cc}$ and is thus a reasonable value for future derivations of two-point calibration lines for CT number to $\mathrm{CaCO}_{3}$ concentration.

Five or more nodules of each size and concentration were obtained. The uniformity of the CT numbers of each set of nodules was verified by scanning each nodule within a water-filled insert at the center of an RMI Model 460 head Quality Control phantom. (Gammex/RMI, Middleton, WI) The RMI phantom is a $19.2 \mathrm{~cm}$ diameter, $7 \mathrm{~cm}$ thick waterequivalent plastic cylinder which has a thin $(4 \mathrm{~mm})$ outer shell of bone-equivalent plastic. It is a reasonable phantom for determining the CT number of an object at the isocenter of the scanner including a moderate amount of beam hardening. The scans of the nodules were performed on a General Electric (GE) LightSpeed VCT 64-slice CT scanner (GE Healthcare, Waukesha, WI) using a technique of $120 \mathrm{kVp}$, $400 \mathrm{~mA}, 0.8 \mathrm{~s}$ rotation time, 0.531:1 pitch, $20 \mathrm{~mm}$ collimation, 32 simultaneous $0.625 \mathrm{~mm}$ slices, $0.6 \mathrm{~mm}$ slice interval for 9.5 and 16 - $\mathrm{mm}$-diameter nodules, $0.3 \mathrm{~mm}$ slice interval for $4.8 \mathrm{~mm}$ nodules, standard reconstruction, and small fieldof-view (FOV). The VCT scanner is the one that was used for the majority of the studies reported in this paper. The scans were repeated three times for reproducibility. The mean CT numbers of the nodules in Hounsfield units (HU) were determined using the automated technique described in Sec. II E.

\section{Effect of air cavity on CT numbers of reference nodules}

A limited study was performed to determine the effect of an air cavity or lung region on the CT number of a nodule. 
For this study, a $9.5 \mathrm{~mm}$ diameter, $50 \mathrm{mg} / \mathrm{cc}$ reference nodule was scanned in three configurations. (1) It was placed within a water-filled cylindrical container that in turn was placed in a $1.8 \mathrm{~cm}$ diameter hole in a tapered solid water insert that was positioned at the center of the RMI Model 460 head phantom. The nodule was thus surrounded completely by liquid or solid water. The RMI phantom was centered within the scan circle and scans were performed using the techniques described below. (2) The same reference nodule was scanned in the $1.8 \mathrm{~cm}$ diameter cylindrical hole in the tapered solid water insert within the RMI phantom. The nodule was positioned on some very low density foam to locate it vertically near the center of the hole. This represented a situation in which the nodule was contained within a $1.8 \mathrm{~cm}$ diameter air cavity. (3) The entire tapered solid water insert was removed from the center of the RMI phantom, leaving a larger air cavity. The nodule was placed on some very low density foam to position it vertically near the center of this larger cavity. The major axis of the cavity was parallel to the CT scan direction, and the shape of the cavity was that of a 3.5 to $5 \mathrm{~cm}$ diameter frustum of a right circular cone. The nodule was located near the middle of this cavity along the scan direction. The diameter of the cavity at this central location was about $4.4 \mathrm{~cm}$, and we will refer to this as the $4.4 \mathrm{~cm}$ diameter air cavity in the remainder of this paper.

The study was performed on a General Electric LightSpeed Ultra-8 CT scanner (GE Healthcare, Waukesha, WI) using a technique of $120 \mathrm{kVp}, 200 \mathrm{~mA}, 1 \mathrm{~s}$ rotation time, 0.625:1 pitch, eight simultaneous $1.25 \mathrm{~mm}$ slices, $0.6 \mathrm{~mm}$ slice interval, standard reconstruction, and small field of view. Later, the study was repeated on the GE LightSpeed VCT 64-slice CT scanner. The technique factors employed on the VCT scanner were $120 \mathrm{kVp}, 400 \mathrm{~mA}, 0.8 \mathrm{~s}$ rotation time, $0.531: 1$ pitch, 32 simultaneous $0.625 \mathrm{~mm}$ slices, $0.3 \mathrm{~mm}$ slice interval, standard reconstruction, and small field of view. Recently, the Cardiology Department at the University of Michigan installed a Siemens Sensation 64 slice CT scanner (Siemens AG, Medical Solutions Erlangen, Germany), and the same study was repeated on that scanner using a technique of $120 \mathrm{kVp}, 200 \mathrm{~mA}, 1 \mathrm{~s}$ rotation time, 0.6:1 pitch, 20 simultaneous $1.2 \mathrm{~mm}$ slice acquisition, $1.5 \mathrm{~mm}$ slice thickness and $1.5 \mathrm{~mm}$ slice interval reconstruction, B30s reconstruction kernel ("B" denotes "body," "s" denotes standard sampling mode, and this is described as a medium soft tissue kernel; to our knowledge, the B30s kernel that we employed is the same as the B30 kernel that ACRIN recommends for Siemens CT scanners in the NLST study), and small field of view. In all cases for this preliminary study of the effect of air cavity size, the CT numbers of the nodules were evaluated manually using regions of interest (ROI) that were placed at the centers of the nodules well within the outer borders, and the maximum of the mean CT numbers within ROIs in two or more adjacent slices through the nodules were chosen as the representative mean CT numbers. Single acquisitions were analyzed for this part of the study as the reproducibility of the representative CT numbers was found to be very good (1.5 HU or less).

\section{Uniformity and reproducibility of CT numbers of nodules in the thorax section}

A study was performed to determine the reproducibility and uniformity of the CT numbers of nodules in the thorax phantom. The reproducibility was investigated by scanning the thorax phantom three times and calculating the mean CT numbers and the standard deviations of the CT numbers in the central regions of the nodules. (See details below.) Uniformity was determined by comparing the mean CT numbers for nodules of the same size and composition at various locations within the lung field. The nodules were placed within a lung simulating foam in the lung region of the thorax phantom for this study. The foam was Rogers Poron 4701-50-15 cellular urethane (Rogers Corporation, Carol Stream, IL) which has a density of $0.24 \mathrm{~g} / \mathrm{cc}$ and a measured CT number of about $-760 \mathrm{HU}$. The density is very similar to the National Bureau of Standards value for lung of $0.26 \mathrm{~g} / \mathrm{cc},{ }^{10}$ and the CT number is similar to that which is observed in patient lungs. ${ }^{11} \mathrm{~A}$ scalpel was used to make slits in the foam for the insertion of the nodules (See Fig. 1). The spherical nodules described in Sec. II B (diameters of 4.8, 9.5, and $16 \mathrm{~mm}$ and $\mathrm{CaCO}_{3}$ concentrations of 50 and $100 \mathrm{mg} / \mathrm{cc}$ ) were also employed in this part of the study. Three scanning protocols were utilized: (1) the protocol recommended for the National Cancer Institute/American College of Radiology Imaging Network (ACRIN) National Lung Screening Trial (NLST), (2) the protocol recommended for the National Heart, Lung and Blood Institute's Lung Tissue Research Consortium study (LTRC), and (3) a high resolution scan protocol that we devised. The latter employed a much smaller pitch, thinner slices, smaller or equivalent slice intervals, and more than twice the mAs of the other techniques. This high resolution scan protocol is referred to as the "gold standard" for the purpose of this study because it was expected to provide better estimates of the true CT numbers of the nodules due to the smaller voxel size and lower noise than the other clinical protocols. Technique factors for the protocols are listed in Table I. All were implemented on a 64-slice GE Lightspeed VCT scanner using $20 \mathrm{~mm}$ rather than $40 \mathrm{~mm}$ total collimation in order to reduce $\mathrm{x}$-ray scatter and minimize potential helical artifacts. To ensure the accuracy of the measured CT numbers, a full calibration of the VCT scanner was performed on the day of our investigation, just prior to the collection of the data. It should be noted that at the time of our study, ACRIN had not yet established recommended technique factors for the 64-slice GE VCT scanner. We therefore chose to employ the technique factors for the 16-slice GE scanners in our investigations. Subsequent to our study, ACRIN published the recommended techniques for the GE VCT scanner, which include the use of lower mAs $(25 \mathrm{mAs}$ vs. $40 \mathrm{mAs}$ for a "small body"), a smaller pitch (0.984:1 vs. $1.375: 1)$, and larger total collimation $(64 \times 0.625 \mathrm{~mm}$ $=40 \mathrm{~mm}$ vs. our $20 \mathrm{~mm}$ ). The final reconstruction slice thickness $(2.5 \mathrm{~mm})$ and slice interval $(2 \mathrm{~mm})$ are the same as we employed. 
TABLE I. X-ray technique factors employed in the CT number reproducibility and uniformity study.

\begin{tabular}{|c|c|c|c|c|c|c|c|}
\hline Protocol & Pitch & $\begin{array}{l}\text { Thickness } \\
(\mathrm{mm})\end{array}$ & $\begin{array}{l}\text { Interval } \\
(\mathrm{mm})\end{array}$ & $\mathrm{kVp}$ & $\mathrm{mAs}$ & $\begin{array}{l}\text { Reconstruction } \\
\text { kernel }\end{array}$ & $\begin{array}{l}\text { FOV } \\
(\mathrm{cm})\end{array}$ \\
\hline $\begin{array}{l}\text { High } \\
\text { Resolution }\end{array}$ & $0.531: 1$ & 0.625 & $0.3 / 0.625^{\mathrm{a}}$ & 120 & 320 & Standard & $30 / 36^{\mathrm{b}}$ \\
\hline NLST & $1.375: 1$ & 2.5 & 2 & 120 & 40 & Standard & 36 \\
\hline LTRC & $1.375: 1$ & 1.25 & 0.625 & 140 & 150 & Bone & 30 \\
\hline
\end{tabular}

${ }^{\mathrm{a}} 0.3 \mathrm{~mm}$ for $4.8-\mathrm{mm}$ diameter nodules and $0.625 \mathrm{~mm}$ for 9.5 and $16-\mathrm{mm}$ diameter nodules.

$\mathrm{b}_{30} \mathrm{~cm}$ for $4.8 \mathrm{~mm}$ diameter nodules and $36 \mathrm{~cm}$ for 9.5 and $16-\mathrm{mm}$ diameter nodules.

\section{E. Automated method for the computation of the mean CT numbers of the nodules}

In preliminary studies, it was found that determining representative mean CT numbers of nodules from manually positioned regions of interest (ROIs) required extreme care in the positioning and sizing of the ROIs, especially for the smaller nodules. In order to avoid these problems, an automated nodule analysis technique was developed. It utilizes a $3 \mathrm{D}$ active contour algorithm developed by our CAD group to segment lung nodules in CT scans. The segmentation is performed in 3D volumetric images formed with isotropic voxels that are created by interpolating the images such that the side lengths of the voxels have a dimension equal to the minimum of either the $x-y$ pixel size or the slice interval. Specifically, for cases in which the pixel size in the $x-y$ plane is smaller than the slice interval in the $z$ direction, linear interpolation is employed in the $z$ direction to create interpolated slice intervals equal to the pixel size. For cases in which the slice interval is smaller than the pixel size, bilinear interpolation of the axial slices is employed to create interpolated pixel sizes equal to the slice interval. The centroid of a nodule is determined as the center of mass of the segmented objects with the CT voxel values as the weighting factors. The centroid serves as the center of spherical ROI that is employed for the calculation of the mean CT number in the nodule. The size of the spherical ROIs was set to a percentage of the segmented nodule volume. A value of $10 \%$ was decided upon to reduce volume averaging yet include a substantial number of voxels in the computation (e.g., for the scanning protocols employed in this study, a $10 \%$ volume corresponded with 19 to 251 voxels for the $4.8 \mathrm{~mm}$ diameter nodules, 147 to 251 voxels for the $9.5 \mathrm{~mm}$ diameter voxels, and 619 to 1021 voxels for the $16 \mathrm{~mm}$ diameter nodules.)

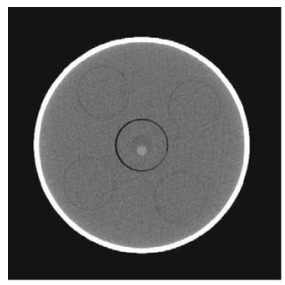

(a)

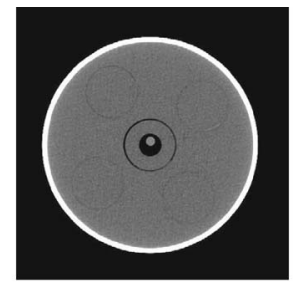

(b)

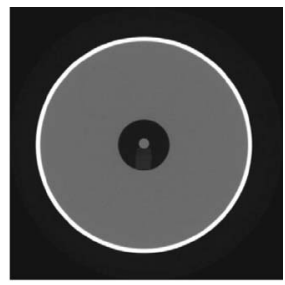

(c)
FIG. 2. Images of $9.5 \mathrm{~mm}$ diameter $50 \mathrm{mg} / \mathrm{cc}$ spherical nodule scanned in RMI QC phantom (a) in water, (b) in a small $1.8 \mathrm{~cm}$ diameter air cavity, and (c) in a larger $4.4 \mathrm{~cm}$ diameter air cavity.
To illustrate the range of number of voxels required, consider the true volume of the $4.8 \mathrm{~mm}$ diameter nodule $V$ $=4 / 3 \pi(2.4 \mathrm{~mm})^{3}=57.9 \mathrm{~mm}^{3}$. The $10 \%$ volume is $5.79 \mathrm{~mm}^{3}$.

For a $36 \mathrm{~cm}$ FOV and a $512 \times 512$ image matrix, the pixel size is $0.703 \times 0.703 \mathrm{~mm}$. If the slice interval is $1.25 \mathrm{~mm}$, then linear interpolation is performed in the $z$ direction to result in isotropic voxels with side lengths of $0.703 \mathrm{~mm}$. Thus, to represent $10 \%$ of the volume, it would require approximately $5.79 \mathrm{~mm}^{3} /(0.703)^{3} \mathrm{~mm}^{3} \approx 17$ voxels. However, if the slice interval is $0.3 \mathrm{~mm}$ and the pixel size is $0.703 \mathrm{~mm}$, then bilinear interpolation is performed on the axial slices to obtain isotropic voxels with side lengths of $0.3 \mathrm{~mm}$. At this voxel size, it would require approximately $5.79 \mathrm{~mm}^{3} /(0.3)^{3} \mathrm{~mm}^{3} \approx 214$ voxels to represent the $10 \%$ volume.

It is interesting to note that although the $10 \%$ volume figure seems small, the radius of the spherical ROI is almost half $(46.4 \%)$ of the radius of the segmented nodule. (The cube root of $10 \%=46.4 \%$.)

Finally, another feature of our nodule segmentation technique is the automated delineation of a lung wall mask which is a boundary that discourages the contour from growing into the lung wall. Both the computer-segmented nodule contours and lung wall mask are displayed as overlays on the original CT images, and manual adjustments can be made to each if the automated segmentation is found to be unsatisfactory.

\section{RESULTS}

\section{A. Automated nodule segmentation}

All segmented contours by the automated method were visually inspected, and it was found that no manual corrections of the nodule contours were necessary. The only manual adjustments required were to the lung wall mask, which occurred in only 12 of 11780 interpolated nodule slices $(0.1 \%)$.

\section{B. CT number uniformity of the sets of reference nodules}

The range of mean CT numbers, overall mean CT numbers, and standard deviations of those mean CT numbers for the sets of reference lung nodules of each type that were scanned in water in the RMI QC phantom on the GE LightSpeed VCT scanner are listed in Table II. Values for the five nodules of each category that yielded the most consistent average CT numbers (minimal overall range of average CT 
TABLE II. Average, standard deviations, and ranges of mean CT numbers (HU) of sets of reference nodules scanned in water in an RMI QC phantom on a GE LightSpeed VCT CT scanner. Results are for 30 nodules (five $4.8 \mathrm{~mm}-50 \mathrm{mg} / \mathrm{cc}$, five $4.8 \mathrm{~mm}-100 \mathrm{mg} / \mathrm{cc}$, etc.), which were each scanned three times. (120 kVp, $320 \mathrm{mAs}$, $0.53: 1$ pitch, $0.625 \mathrm{~mm}$ slice, $0.3 \mathrm{~mm}$ slice interval for $4.8 \mathrm{~mm}$ nodules, $0.625 \mathrm{~mm}$ slice interval for others, $25 \mathrm{~cm}$ FOV).

\begin{tabular}{lcrrr}
\hline \hline Concentration & & $\begin{array}{c}4.8 \mathrm{~mm} \\
\text { nodules }\end{array}$ & $\begin{array}{c}9.5 \mathrm{~mm} \\
\text { nodules }\end{array}$ & $\begin{array}{r}16 \mathrm{~mm} \\
\text { nodules }\end{array}$ \\
\hline \multirow{3}{*}{$50 \mathrm{mg} / \mathrm{cc}$} & Minimum mean CT no. & 53.1 & 61.5 & 59.4 \\
& Maximum Mean CT no. & 58.0 & 64.5 & 60.1 \\
& Range (max-min) & 4.8 & 3.0 & 0.8 \\
& Average mean CT no. & 56.0 & 63.1 & 59.7 \\
& Standard deviation & 2.0 & 1.3 & 0.3 \\
$100 \mathrm{mg} / \mathrm{cc}$ & Minimum mean CT no. & 102.3 & 111.9 & 112.0 \\
& Maximum mean CT no. & 108.9 & 113.4 & 112.3 \\
& Range (max-min) & 6.7 & 1.6 & 0.4 \\
& Average mean CT no. & 104.7 & 112.5 & 112.2 \\
& Standard deviation & 2.7 & 0.6 & 0.2 \\
\hline \hline
\end{tabular}

numbers) are displayed. The remaining nodules of each category were considered outliers or defective and were not used in further studies.

\section{Effect of lung/air cavity size on measured CT number of nodule}

Images of the $9.5 \mathrm{~mm}$ diameter, $50 \mathrm{mg} / \mathrm{cc} \mathrm{CaCO}_{3}$ reference nodule that was scanned near the center of the RMI phantom within water and two different sized air cavities are shown in Fig. 2.

The measured CT numbers of this nodule on three different CT scanners are listed in Table III.

\section{CT numbers of nodules in lung sections of thorax phantom}

The mean CT numbers and standard deviations of the 50 and $100 \mathrm{mg} / \mathrm{cc}$ reference nodules at various locations within the thorax section for three CT scans obtained with each technique are listed in Tables IV-VI. The specific locations of the nodules are shown in Fig. 3.

\section{DISCUSSION}

\section{A. Measured CT numbers of reference nodules in water}

The CT numbers of the 50 and the $100 \mathrm{mg} / \mathrm{cc}^{\mathrm{CaCO}_{3}}$ reference nodules measured in water at the isocenter of the scanner (Table II) are close to the 59 and $118 \mathrm{HU}$, respec- tively, estimated for $\mathrm{x}$-ray beams with effective energies of about $70 \mathrm{keV}$. The measured CT numbers are fairly independent of size for the larger $(9.5$ and $16 \mathrm{~cm})$ nodules. However, they are consistently lower by about 7.7 and $5.4 \mathrm{HU}$ for the $4.8 \mathrm{~mm}$ diameter, 100 and $50 \mathrm{mg} / \mathrm{cc}$ nodules, respectively. This is likely due to a greater proportion of the voxels within the $4.8 \mathrm{~mm}$ diameter nodules being affected by volume averaging with the surrounding lower attenuation water. The relative amount of volume averaging for different size spherical nodules is directly related to the ratio of the surface area of the sphere that is in contact with the water divided by the volume of the sphere, and this ratio is inversely proportional to the sphere diameter. The effect of the volume averaging is further enhanced especially for the small nodules by the use of a standard CT reconstruction kernel. This kernel produces smooth-looking CT images and, in doing so, tends to increase the relative size of the zone surrounding the nodule that impacts the CT numbers within the nodule. Another possible reason for the decreased $\mathrm{CT}$ numbers of the small nodules might be that those nodules had lower $\mathrm{CaCO}_{3}$ concentrations. However, this is unlikely as the manufacturer of the nodules, CIRS, informed us that all size balls of a given concentration were constructed from the same batch of $\mathrm{CaCO}_{3}$ in epoxy resin. Furthermore, the same decreased CT numbers for smaller nodules were observed when, in a separate study, we employed techniques identical to those for the $\mathrm{CaCO}_{3}$ nodules to scan solid, uniform acrylic balls of different sizes within liquid water in the RMI phantom. The aver-

TABLE III. Measured CT numbers (HU) of a $9.5 \mathrm{~mm}$ diameter, $50 \mathrm{mg} / \mathrm{cc} \mathrm{CaCO}_{3}$ nodule when scanned in water, in a $1.8 \mathrm{~cm}$ diameter air cavity, and in a $4.4 \mathrm{~cm}$ diameter air cavity for $3 \mathrm{CT}$ scanners.

\begin{tabular}{lccc}
\hline \hline \multicolumn{1}{c}{ Scanner } & CT no. in water & $\begin{array}{c}\text { CT no. in } 1.8 \mathrm{~cm} \\
\text { diameter air cavity }\end{array}$ & $\begin{array}{c}\text { CT no. in } 4.4 \mathrm{~cm} \\
\text { diameter air cavity }\end{array}$ \\
\hline GE LightSpeed Ultra-8 & 66 & 51 & 36 \\
GE LightSpeed VCT & 64 & 37 & 19 \\
Siemens Sensation 64 & 66 & 99 & 95 \\
\hline \hline
\end{tabular}


TABLE IV. Mean CT numbers (HU) of the $4.8 \mathrm{~mm}$ diameter reference nodules for three scans at each technique and the standard deviations of the mean CT numbers.

\begin{tabular}{|c|c|c|c|c|c|c|c|}
\hline & \multirow[b]{2}{*}{$\begin{array}{c}\text { Nodule } \\
\text { no. }\end{array}$} & \multicolumn{2}{|c|}{ High resolution } & \multicolumn{2}{|r|}{ NLST } & \multicolumn{2}{|r|}{ LTRC } \\
\hline & & Mean & Standard deviation & Mean & Standard deviation & Mean & Standard deviation \\
\hline \multirow{5}{*}{$\begin{array}{c}100 \\
\mathrm{mg} / \mathrm{cc}\end{array}$} & 1 & 47.5 & 0.4 & -88.3 & 11.8 & 74.1 & 12.6 \\
\hline & 2 & 44.3 & 2.1 & -78.7 & 20.4 & 73.5 & 6.9 \\
\hline & 3 & 44.3 & 2.2 & -68.2 & 7.8 & 72.7 & 12.2 \\
\hline & 4 & 42.0 & 0.6 & -66.2 & 6.6 & 60.2 & 0.6 \\
\hline & 5 & 40.7 & 2.4 & -72.6 & 10.5 & 70.9 & 7.4 \\
\hline \multicolumn{2}{|c|}{ Overall mean } & \multicolumn{2}{|r|}{$43.8 \pm 2.6$} & \multicolumn{2}{|r|}{$-74.8 \pm 9.0$} & \multicolumn{2}{|r|}{$70.3 \pm 5.7$} \\
\hline 50 & 6 & -2.8 & 1.5 & -116.5 & 0.3 & 15.2 & 3.5 \\
\hline \multirow[t]{4}{*}{$\mathrm{mg} / \mathrm{cc}$} & 7 & -8.0 & 2.2 & -127.1 & 4.6 & 8.6 & 2.3 \\
\hline & 8 & -10.2 & 0.4 & -149.1 & 22.7 & 2.2 & 6.0 \\
\hline & 9 & -8.6 & 2.2 & -141.1 & 2.0 & 17.7 & 0.6 \\
\hline & 10 & -8.9 & 0.8 & -157.2 & 4.7 & 12.7 & 1.6 \\
\hline \multicolumn{2}{|c|}{ Overall mean } & \multicolumn{2}{|r|}{$-7.7 \pm 2.8$} & \multicolumn{2}{|c|}{$-138.2 \pm 16.4$} & \multicolumn{2}{|r|}{$11.3 \pm 6.1$} \\
\hline
\end{tabular}

age CT numbers of acrylic balls of 3.2, 6.2, and $12.7 \mathrm{~mm}$ diameter as determined with the automated software in $10 \%$ volumes were 107.0, 119.2, and 119.1 $\mathrm{HU}$, respectively. It should be noted that the decrease in average CT number for the smaller nodules cannot be explained by beam hardening or scatter since these would result in lower CT numbers in the larger rather than the smaller nodules. A list of expected CT numbers as a function of $\mathrm{X}$-ray energy that was computed using the XCOM program, assuming the mass density of pure $\mathrm{CaCO}_{3}$ is $2.93 \mathrm{~g} / \mathrm{cc},{ }^{12}$ is displayed in Table VII.

\section{B. Effect of an air cavity on the measured CT numbers}

The dependence of the measured CT numbers of the reference nodules on their surroundings (e.g., water or air) is of concern. When the surroundings change from water to $4.4 \mathrm{~cm}$ of air (Table III), the CT number of the $9.5 \mathrm{~mm}$ diameter nodule decreased by $30 \mathrm{HU}$ for the GE LightSpeed Ultra-8, decreased by $45 \mathrm{HU}$ for the GE LightSpeed VCT, and increased by $29 \mathrm{HU}$ for the Siemens Sensation 64 . Tarver $e t a l^{2}$ and others observed similar effects with earlier CT scanners- the CT number of simulated nodules or dense solutions increasing with increasing environmental density on some scanners and decreasing on others. Tarver et al.'s explanation for this phenomenon is that "scanner-dependent beam hardening corrections (or perhaps other aspects of image reconstruction) are crucial to the magnitude and direction of the environmental density artifact." ${ }^{2}$ It is believed that the decrease in CT number with decreased environmental density (i.e., air surrounding) that was observed with the GE

TABLE V. Mean CT numbers (HU) of the $9.5 \mathrm{~mm}$-diameter reference nodules for 3 scans at each technique and the standard deviations of the mean CT numbers.

\begin{tabular}{|c|c|c|c|c|c|c|c|}
\hline & \multirow{2}{*}{$\begin{array}{c}\text { Nodule } \\
\text { no. }\end{array}$} & \multicolumn{2}{|c|}{ High Resolution } & \multicolumn{2}{|r|}{ NLST } & \multicolumn{2}{|r|}{ LTRC } \\
\hline & & Mean & Standard deviation & Mean & Standard deviation & Mean & Standard deviation \\
\hline \multirow{5}{*}{$\begin{array}{c}100 \\
\mathrm{mg} / \mathrm{cc}\end{array}$} & 1 & 75.5 & 0.4 & 68.6 & 1.4 & 85.3 & 0.4 \\
\hline & 2 & 74.5 & 1.1 & 64.3 & 3.1 & 86.8 & 2.2 \\
\hline & 3 & 72.0 & 0.9 & 64.0 & 2.1 & 82.3 & 1.5 \\
\hline & 4 & 64.5 & 1.4 & 57.5 & 0.8 & 75.4 & 1.7 \\
\hline & 5 & 64.3 & 0.3 & 55.4 & 4.2 & 78.3 & 3.0 \\
\hline \multicolumn{2}{|c|}{ Overall mean } & \multicolumn{2}{|r|}{$70.2 \pm 5.4$} & \multicolumn{2}{|r|}{$62.0 \pm 5.4$} & \multicolumn{2}{|r|}{$81.6 \pm 4.8$} \\
\hline & 6 & 18.4 & 0.3 & 10.5 & 1.0 & 33.4 & 1.9 \\
\hline & 7 & 14.4 & 0.3 & 8.5 & 3.7 & 28.6 & 0.9 \\
\hline 50 & 8 & 13.1 & 0.1 & 2.6 & 0.3 & 25.9 & 2.6 \\
\hline & 9 & 14.8 & 1.9 & 14.1 & 1.1 & 34.3 & 0.5 \\
\hline & 10 & 23.0 & 0.3 & 17.6 & 2.5 & 40.7 & 1.6 \\
\hline Over & Mean & \multicolumn{2}{|r|}{$16.7 \pm 4.0$} & \multicolumn{2}{|r|}{$10.7 \pm 5.7$} & \multicolumn{2}{|r|}{$32.6 \pm 5.7$} \\
\hline
\end{tabular}


TABLE VI. Mean CT numbers (HU) of the $16 \mathrm{~mm}$ diameter reference nodules for three scans at each technique and the standard deviations of the mean CT numbers.

\begin{tabular}{|c|c|c|c|c|c|c|c|}
\hline & \multirow[b]{2}{*}{$\begin{array}{c}\text { Nodule } \\
\text { no. }\end{array}$} & \multicolumn{2}{|c|}{ High resolution } & \multicolumn{2}{|r|}{ NLST } & \multicolumn{2}{|r|}{ LTRC } \\
\hline & & Mean & Standard deviation & Mean & Standard deviation & Mean & Standard deviation \\
\hline \multirow{5}{*}{$\begin{array}{c}100 \\
\mathrm{mg} / \mathrm{cc}\end{array}$} & 1 & 81.1 & 0.6 & 82.2 & 1.6 & 83.0 & 1.5 \\
\hline & 2 & 79.4 & 0.4 & 79.9 & 1.5 & 82.6 & 2.3 \\
\hline & 3 & 79.1 & 0.3 & 78.4 & 1.9 & 82.5 & 1.3 \\
\hline & 4 & 68.4 & 0.3 & 69.2 & 0.4 & 75.0 & 0.9 \\
\hline & 5 & 78.9 & 0.5 & 81.9 & 0.7 & 83.4 & 1.5 \\
\hline \multicolumn{2}{|c|}{ Overall mean } & \multicolumn{2}{|r|}{$77.4 \pm 5.1$} & \multicolumn{2}{|r|}{$78.3 \pm 5.3$} & \multicolumn{2}{|r|}{$81.3 \pm 3.5$} \\
\hline \multirow{5}{*}{$50 \mathrm{mg} / \mathrm{cc}$} & 6 & 25.1 & 0.4 & 25.6 & 3.0 & 33.9 & 1.9 \\
\hline & 7 & 20.3 & 0.2 & 22.4 & 2.0 & 29.1 & 0.9 \\
\hline & 8 & 19.3 & 0.2 & 19.6 & 3.7 & 27.9 & 0.5 \\
\hline & 9 & 25.2 & 0.2 & 25.5 & 1.5 & 33.5 & 1.3 \\
\hline & 10 & 25.5 & 0.6 & 26.3 & 0.3 & 33.4 & 0.4 \\
\hline \multicolumn{2}{|c|}{ Overall mean } & \multicolumn{2}{|r|}{$23.1 \pm 3.0$} & \multicolumn{2}{|r|}{$23.1 \pm 2.8$} & \multicolumn{2}{|r|}{$31.6 \pm 2.8$} \\
\hline
\end{tabular}

scanners in our study is due to an assumption made in the scanner beam hardening correction algorithm that the entire "patient" is composed of water. The apparent increased x-ray transmission that is present in the projections when the nodules are surrounded by air instead of water translates to a decreased linear attenuation coefficient and a decreased CT number. The opposite increase in the CT number of the nodule when surrounded by air for the Siemens CT scanner is likely an overcompensation of the Siemens beam hardening correction for the expected decrease in CT number at the center of an object. These results tend to indicate that as with early CT scanners, the CT numbers of modern scanners are not necessarily accurate, and the $\mathrm{CT}$ numbers for different scanners can vary significantly. This is of major importance for longitudinal studies in which patients are scanned year after year and for followup studies that may be performed at

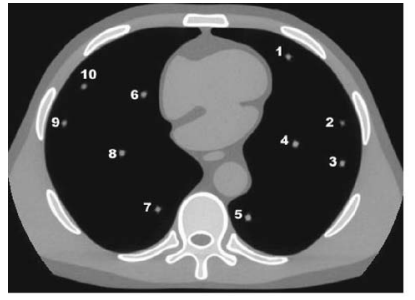

(a)

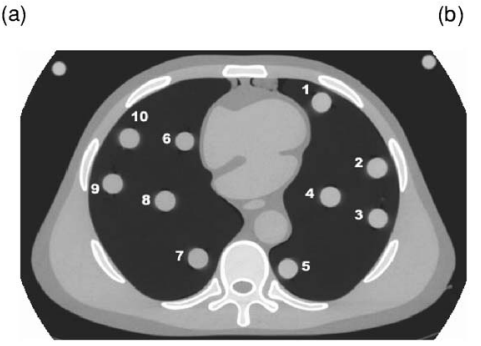

(c)

FIG. 3. CT images of the phantom showing the locations of the individual (a) $4.8 \mathrm{~mm}$ diameter, (b) $9.5 \mathrm{~mm}$ diameter, and (c) $16 \mathrm{~mm}$ diameter simulated lung nodules.
3,6 , or 12 month intervals to detect changes in the size and composition of detected lung nodules. It is also relevant in the determination of whether nodules are calcified based on their measured CT numbers. For such measurements, the overestimates of the CT numbers that were obtained with the Siemens scanner could result in noncalcified nodules being called calcified and hence an increase in false negatives. Conversely, the underestimates of the CT numbers obtained with the GE scanners could result in calcified nodules being called noncalcified and hence an increase in false positives. To ensure consistency in the CT numbers of the nodules, it is advised that the scans be performed each time on the same make and model scanner. Our results also point to the need to develop CT number correction factors to relate CT numbers from one scanner to those from another. The patient reference phantoms like the ones used in the present study can be used for this purpose. These factors also need to be accounted for in the development of CAD features. For example, one group has proposed using the fraction of the number of voxels greater than $150 \mathrm{HU}$ (indicating significant

TABLE VII. CT numbers of $50 \mathrm{mg} / \mathrm{cc} \mathrm{CaCO}_{3}$ and $100 \mathrm{mg} / \mathrm{cc} \mathrm{CaCO}_{3}$ in water mixtures for monoenergetic $\mathrm{x}$-ray beams.

\begin{tabular}{|c|c|c|}
\hline Energy (keV) & $\begin{array}{c}\text { CT no. } \\
50 \mathrm{mg} / \mathrm{cc} \mathrm{CaCO}_{3}\end{array}$ & $\begin{array}{c}\text { CT no. } \\
100 \mathrm{mg} / \mathrm{cc} \mathrm{CaCO}_{3}\end{array}$ \\
\hline 60 & 74.2 & 148.5 \\
\hline 62 & 70.6 & 141.1 \\
\hline 64 & 67.2 & 134.5 \\
\hline 66 & 64.3 & 128.5 \\
\hline 68 & 61.6 & 123.1 \\
\hline 70 & 59.1 & 118.3 \\
\hline 72 & 56.9 & 113.9 \\
\hline 74 & 54.9 & 109.9 \\
\hline 76 & 53.1 & 106.3 \\
\hline 78 & 51.5 & 102.9 \\
\hline 80 & 50.0 & 99.9 \\
\hline
\end{tabular}


calcium) and the fraction of the number of voxels less than 0 (indicating significant calcium and fat) as features. ${ }^{13}$ The CT numbers themselves and therefore the CT number thresholds will likely be different on different $\mathrm{CT}$ scanners and may differ with nodule size, lung size, and the size of the patient. Finally, the effect of lung size on CT number accuracy is especially relevant to the LTRC study in which the volumetric CT scan protocol calls for the acquisition of three sets of images - one set obtained with the patient supine at full inspiration, a second set with the patient supine at suspended full expiration, and a third with the patient prone at full inspiration (www.ltrcpublic.com/images.htm). Based on our results, the CT number of a given nodule may vary considerably between full inspiration and full expiration due to changes in the size of the lungs.

\section{Dependence of CT number on nodule size in the anthropomorphic phantom}

When the simulated lung nodules were scanned within the lung regions of the anthropomorphic phantom, there was considerably greater dependence of the measured mean CT numbers of the nodules on their size than when the same nodules were scanned at the center of a water-equivalent phantom. For example, the mean CT numbers of the $100 \mathrm{mg} / \mathrm{cc} \mathrm{CaCO}_{3}$ nodules in the lung region of the anthropomorphic phantom for the high resolution CT scanning technique (Tables IV-VI) were 43.8, 70.2, and 77.4 HU for the $4.8,9.5$, and $16 \mathrm{~mm}$ diameter nodules, respectively. Corresponding values in water (Table II) were 104.7, 112.5, and 112.2 HU. The large variation in CT number with size of the spherical nodule in the anthropomorphic phantom that we observed is similar to what was found in previous studies using cylindrical nodules with single slice axial scanners. ${ }^{4}$ The values are more consistent for the larger 9.5 and $16 \mathrm{~mm}$ nodules than with the early scanners, but there is still a large decrease in CT number for the smaller $4.8 \mathrm{~mm}$ diameter nodules. In comparing the results for the $9.5 \mathrm{~mm}$ diameter (Table $\mathrm{V}$ ) and $16 \mathrm{~mm}$ diameter nodules (Table VI) with both the high resolution and NLST techniques, one finds that, on average, the CT numbers are lower for the $9.5 \mathrm{~mm}$ diameter nodules than for the $16 \mathrm{~mm}$ diameter nodules. This is different from what was observed when the same nodules were scanned in water (Table II) (CT numbers about the same for both sizes) and the opposite of what would be expected due to increased beam hardening within the larger $16 \mathrm{~mm}$ diameter nodules. However, it is consistent with the results obtained by Zerhouni et al. in an anthropomorphic lung section with a GE $9800 \mathrm{CT}$ scanner that the larger the reference nodule, the larger the CT number. ${ }^{4}$ When the results for the sets of different size nodules are compared using analysis of variance (ANOVA) (two-factor ANOVA with replication to account for the repeated studies), it is found that for all techniques (high resolution, NLST and LTRC) one can reject the null hypothesis (that the mean CT numbers for the three different sized reference nodules are the same) at a level of significance of 0.00001 or less. More detailed analysis on a paired basis (e.g., for a given calcium concentration, all
$4.8 \mathrm{~mm}$ vs. all $9.5 \mathrm{~mm}$, all $4.8 \mathrm{~mm}$ vs. all $16 \mathrm{~mm}$, all $9.5 \mathrm{~mm}$ vs. all $16 \mathrm{~mm}$ ) using two-sample two-tailed $t$ tests indicates that in almost all cases one can reject the null hypothesis that there is no difference in mean CT number due to nodule size, with $p$ values less than 0.0004 . The only exceptions are for the $9.5 \mathrm{~mm}$ vs. $16 \mathrm{~mm}$ nodules with the LTRC technique for which the $p$ values were 0.84 and 0.52 for the 100 and $50 \mathrm{mg} / \mathrm{cc}$ nodules, respectively. For only this very limited set, one cannot reject the null hypothesis that there is no difference in CT numbers due to nodule size. Finally, the nearly universal variability in mean CT number with nodule size that was observed in this study confirms the need to obtain separate calibrations for different nodule sizes in a standardized reference phantom method For example, one might employ three size (diameter) categories-small $(\sim 5 \mathrm{~mm})$, medium $(\sim 10 \mathrm{~mm})$, and large $(\sim 15 \mathrm{~mm})$.

\section{Reproducibility of measured CT numbers}

In general, the reproducibility of the measured CT numbers is best for the larger simulated nodules and for the higher $z$ resolution (thinner slice, smaller slice interval) CT scans. The average of the standard deviations of the measured CT numbers of the ten $16 \mathrm{~mm}$ nodules (Table VI) for the repeated scans was $0.37 \mathrm{HU}$ for the high resolution technique, 1.66 HU for the NLST technique, and 1.26 HU for the LTRC technique. Corresponding values for the complete set of $9.5 \mathrm{~mm}$ nodules (Table V) were 0.70, 2.02, and $1.62 \mathrm{HU}$. Those for the $4.8 \mathrm{~mm}$ nodules (Table IV) were 1.47, 9.13, and 5.37 HU. Thus, in this controlled phantom experiment, excellent short-term reproducibility of about $2 \mathrm{HU}$ or less was achieved for all nodule sizes with the high resolution technique and for all except the $4.8 \mathrm{~mm}$ diameter nodules with the NLST and LTRC techniques. It is important to note that the long-tern reproducibility would be expected to be worse in longitudinal patient studies due to greater variability in patient positioning, in x-ray scanner calibration status, in degree of lung inspiration or expiration, in patient motion, and possibly in patient body size (e.g., due to gain or loss of weight). The anthropomorphic phantom in our experiment was scanned at exactly the same table height each time, and the phantom was not moved on the table (no change in lateral position). Such consistency in setup would not be the case in longitudinal patient scans. Since the CT scanners employ a bow-tie filter to shape the incident $\mathrm{x}$-ray beam intensity in order to compensate for greater attenuation at the center than at the periphery of the patient, any change in patient position will affect the x-ray energy spectrum at locations within the patient, which will in turn affect the measured CT numbers. In the phantom experiment, there was some variability in helical data interpolation due to the changes in the starting angle of the x-ray tube in the helical acquisition. The starting angle is a parameter that cannot be controlled by the user. The effect of the starting angle should be greater for techniques that employ larger pitches (NLST and LTRC) because such pitches result in greater differences in the helical projections that are interpolated to reconstruct axial slices. Differences in the $\mathrm{x}$-ray tube starting angle 
TABLE VIII. Mean CT numbers of the $4.8 \mathrm{~mm}$ diameter reference nodules for the high resolution, the modified $1.25 \mathrm{~mm}$ slice NLST, and the standard NLST techniques.

\begin{tabular}{|c|c|c|c|c|c|c|c|}
\hline & \multirow[b]{2}{*}{$\begin{array}{c}\text { Nodule } \\
\text { no. }\end{array}$} & \multicolumn{2}{|c|}{ High resolution } & \multicolumn{2}{|c|}{$1.25 \mathrm{~mm}$ slice NLST } & \multicolumn{2}{|c|}{ Standard NLST } \\
\hline & & Mean & Standard deviation & Mean & Standard deviation & Mean & Standard deviation \\
\hline \multirow{5}{*}{$\begin{array}{c}100 \\
\mathrm{mg} / \mathrm{cc}\end{array}$} & 1 & 47.5 & 0.4 & 39.8 & 9.5 & -88.3 & 11.8 \\
\hline & 2 & 44.3 & 2.1 & 43.4 & 7.1 & -78.7 & 20.4 \\
\hline & 3 & 44.3 & 2.2 & 41.6 & 7.0 & -68.2 & 7.8 \\
\hline & 4 & 42.0 & 0.6 & 33.0 & 7.6 & -66.2 & 6.6 \\
\hline & 5 & 40.7 & 2.4 & 45.0 & 7.6 & -72.6 & 10.5 \\
\hline \multicolumn{2}{|c|}{ Overall mean } & \multicolumn{2}{|r|}{$43.8 \pm 2.6$} & \multicolumn{2}{|c|}{$40.6 \pm 4.6$} & \multicolumn{2}{|c|}{$-74.8 \pm 9.0$} \\
\hline 50 & 6 & -2.8 & 1.5 & -4.4 & 2.5 & -116.5 & 0.3 \\
\hline \multirow[t]{4}{*}{$\mathrm{mg} / \mathrm{cc}$} & 7 & -8.0 & 2.2 & -18.9 & 7.4 & -127.1 & 4.6 \\
\hline & 8 & -10.2 & 0.4 & -37.8 & 4.8 & -149.1 & 22.7 \\
\hline & 9 & -8.6 & 2.2 & -20.5 & 2.0 & -141.1 & 2.0 \\
\hline & 10 & -8.9 & 0.8 & -23.0 & 7.0 & -157.2 & 4.7 \\
\hline \multicolumn{2}{|c|}{ Overall mean } & \multicolumn{2}{|r|}{$-7.7 \pm 2.8$} & \multicolumn{2}{|c|}{$-20.7 \pm 11.6$} & \multicolumn{2}{|c|}{$-138.2 \pm 16.4$} \\
\hline
\end{tabular}

would be expected to have a more deleterious effect on reproducibility in patient studies because the increased heterogeneity of patients compared to phantoms will result in greater changes in the helical projections.

\section{E. Inaccuracy of NLST technique for small nodules}

One of the most important results of this study is that it demonstrates the inaccuracy and unreliability of the current National Lung Screening Trial (NLST) protocol for characterizing the CT numbers of small (5 $\mathrm{mm}$ diameter or less) nodules. The $2.5 \mathrm{~mm}$ slice thickness and $2 \mathrm{~mm}$ slice interval are too large to properly sample small nodules, and the large 1.375:1 pitch does not help. The mean CT number of the five $4.8 \mathrm{~mm}$ diameter $100 \mathrm{mg} / \mathrm{cc}$ nodules (Table IV) was $-75 \mathrm{HU}$ for the NLST technique as compared to $+44 \mathrm{HU}$ for the high resolution $(0.625 \mathrm{~mm}$ thickness, $0.3 \mathrm{~mm}$ slice interval, 0.531:1 pitch) "gold standard" technique, and the mean CT number of the five $4.8 \mathrm{~mm}$ diameter $50 \mathrm{mg} / \mathrm{cc}$ nodules was $-138 \mathrm{HU}$ for the NLST technique as compared to $-8 \mathrm{HU}$ for the high resolution technique Thus, scanning with the NLST technique could result in lung nodules containing as much as $100 \mathrm{mg} / \mathrm{cc} \mathrm{CaCO}_{3}$ appearing to consist of almost pure fat (CT number of about $-100 \mathrm{HU}$ ). Furthermore, the reproducibilities of the mean $\mathrm{CT}$ numbers of the individual nodules for the NLST technique, as represented by the standard deviations for three separate scans of the nodules in the anthropomorphic phantom, were as large as $23 \mathrm{HU}$ (Table IV, NLST, nodule 8) vs. the largest standard deviation of only $2.4 \mathrm{HU}$ for the high resolution technique (Table IV, high resolution, nodule 5).

It is important and interesting to note that for the NLST technique on a 16-slice GE CT scanner, the original helical projections are acquired using a $16 \times 1.25 \mathrm{~mm}(20 \mathrm{~cm}$ total collimation) detector configuration, and this is essentially what we employed. With this configuration, images with a minimum slice thickness of $1.25 \mathrm{~mm}$ can be reconstructed from the original projections. In fact, such additional (retro- spective) reconstructions are performed at some sites such as ours, not for the official NLST readings, but for future clinical readings to assist the radiologists in finding calcifications within small nodules. To investigate the effect of reconstructing the NLST projections to thinner slices, we performed additional reconstructions with $1.25 \mathrm{~mm}$ slice thickness and $1.25 \mathrm{~mm}$ slice interval. We call these the " $1.25 \mathrm{~mm}$ slice NLST" reconstructions. The resulting mean CT numbers for the $1.25 \mathrm{~mm}$ slice NLST technique are much closer to those of the high resolution technique and overall, they are also more reproducible than those for the standard NLST technique. The values are compared in Table VIII.

Based on these results, more accurate CT numbers of small lung nodules than those for the standard NLST technique could be obtained from additional thinner (e.g., $1.25 \mathrm{~mm}$ ) slice reconstructions for all 8- and 16-slice CT scanners that acquire data using multiple $1.25 \mathrm{~mm}$ detector configurations This, however, would not be true for fourslice GE CT scanners for which a $4 \times 2.5 \mathrm{~mm}$ detector configuration is employed, which results in a thinnest reconstruction slice of $2.5 \mathrm{~mm}$, which is the same as the NLST slice thicknesses. Similar conclusions are likely for other manufacturer's CT scanners that are employed in the NLST study with some exceptions [e.g., the Siemens VZ and the Philips MX8000 CT scanners are listed in ACRIN/NLST CT Technique Comparison Chart as being operable in both "4 simultaneous $1 \mathrm{~mm}$ slice" and "4 simultaneous $2.5 \mathrm{~mm}$ slice" modes, where only the former (4 simultaneous $1 \mathrm{~mm}$ slice) mode would be expected to yield improved results for additional reconstructions].

Finally, although the NLST technique results in poor characterization of the CT numbers of the smaller nodules, it may or may not impede the detection of those nodules. The reason is that the CT numbers of the small calcified nodules (e.g., $-138 \mathrm{HU}$ for $50 \mathrm{mg} / \mathrm{cc}$ and $-75 \mathrm{HU}$ for $100 \mathrm{mg} / \mathrm{cc}$ ) are still considerably greater than the average lung parenchyma CT number of about -760 to $-860 \mathrm{HU}^{10}$ If in- 
creased image storage space and increased radiologist read times are not an issue, additional thinner-slice and smaller slice-interval reconstructions should be performed if possible from the original NLST helical projections for improved CT number accuracy and nodule characterization. Alternatively, similar improvements can be attained if small nodules that are detected with the standard NLST technique are later rescanned using a higher resolution technique. A disadvantage of this alternative is that the rescanning of the patient involves additional radiation dose, whereas the retrospective reconstructions are achieved without additional dose.

\section{F. The LTRC technique}

The LTRC scanning technique has two primary differences from the high resolution and NLST techniques-it employs a higher $\mathrm{kVp}(140 \mathrm{kVp}$ vs. $120 \mathrm{kVp})$ and it employs a bone reconstruction kernel instead of a standard reconstruction kernel. The higher $\mathrm{kVp}$ would be expected to result in a higher effective $\mathrm{x}$-ray beam energy and therefore lower linear x-ray attenuation coefficients and lower CT numbers. This, however, is countered by the bone reconstruction kernel which enhances edges and would be expected to increase the CT numbers. As shown in Tables IV-VI, the mean CT numbers of the reference nodules of all three sizes are more consistent with the LTRC technique. This is especially true for the $100 \mathrm{mg} / \mathrm{cc}$ nodules for which the overall mean CT numbers are 70.3, 81.6, and $81.3 \mathrm{HU}$ for the 4.8, 9.5, and $16 \mathrm{~mm}$ diameter nodules, respectively. These values are even more consistent than what should have been the gold standard-the high resolution technique, which has corresponding values of 40.7, 70.2, and 77.4 HU. If one compares the results (not shown in tables) for post-reconstruction images generated from the projections acquired for the LTRC technique but with thinner slices and the standard rather than the bone reconstruction kernel $(140 \mathrm{kVp}, 0.625 \mathrm{~mm}$ thickness, $0.625 \mathrm{~mm}$ slice interval, standard reconstruction, and 1.375:1 pitch), the overall mean CT numbers $(43.7 \pm 3.6$, $63.9 \pm 5.9$, and $70.9 \pm 4.7 \mathrm{HU}$ ) are more similar to those of the high resolution technique. Thus, the bone kernel must be responsible for improving the consistency of the $\mathrm{CT}$ numbers for different size nodules. The increased CT numbers of the $4.8 \mathrm{~mm}$ nodules that are obtained with the bone reconstruction kernel employed in the LTRC technique are a result of the improved resolution (narrower point spread function) associated with that kernel, which in turn reduces volume averaging between the nodule and the adjacent foam/air. While such improvements in CT number consistency may be true for the homogeneous nodules in this study, the results may be very different for heterogenous lung nodules containing clumps of calcifications. This is worthy of further investigation. Also, it should be noted that the bone kernel increases the perceived noise in CT images. This is especially relevant in screening trials like the NLST in which low radiation dose is employed [the CTDIvol for the NLST study ( $2.7 \mathrm{mGy})$ is about 6.4 times less than the CTDIvol for the non-screening LTRC study (17.3 mGy)]. The resulting low-dose CT images will inherently exhibit high quantum mottle, and the bone kernel would make the images even noisier, which could reduce the conspicuity of nodules. The effect of the trade-off between increased CT number and increased noise on nodule detectability needs to be studied if the bone kernel is considered for a screening protocol. Finally, as discussed above, the LTRC three-phase CT scan protocol includes three sets of images, two sets at full inspiration, and one at full expiration. Based on our studies of simulated nodules within air cavities of various sizes in a water-equivalent phantom (II), the CT number of an individual nodule in a patient may differ considerably on some CT scanners between full inspiration and full expiration. This would especially be true if the nodule were located in a part of the lung near the diaphragm where the lung cross-sectional area in the CT scan changes from very small (e.g., about $2 \mathrm{~cm}$ ) to twice as large or greater. It is interesting to note that although there was a large change in the mean CT number of the $9.5 \mathrm{~mm}$ diameter, $50 \mathrm{mg} / \mathrm{cc}$ lung nodule between the $1.8 \mathrm{~cm}$ diameter air cavity situation ( $37 \mathrm{HU})$ and the $4.4 \mathrm{~cm}$ diameter air cavity situation (19 HU) (Table III), the CT number did not change appreciably between the $4.4 \mathrm{~cm}$ air cavity situation $(19 \mathrm{HU})$ and the full lung situation (16.7 HU) (Table V). Thus, the change in lung size with inspiration and expiration may not have a large effect on the CT number of nodules in the majority of the lung, but this is worthy of further investigation.

\section{G. Variation in mean CT number with position in lung}

From the data in Tables IV-VI, it is apparent that, in general, the mean CT numbers of the nodules near the centers of the lungs (positions 4 and 8) and near the spine (positions 5 and 7) tend to be lower than the CT numbers of the nodules at other locations. This can be explained by the increased beam hardening and scatter in these regions. The differences are relatively small (e.g., about 2-11 HU), but for the 9.5 and $16 \mathrm{~mm}$ nodule cases they are greater than the consistency ranges of the mean CT numbers of the sets of reference nodules of each type when scanned at the isocenter in water ( $\sim 2$ to $3 \mathrm{HU})$ (Table II). Thus, the differences are likely to be real. Since the differences are relatively small, the variability in CT number with position within the lung field may not be as important an issue with modern CT scanners as it was with early CT scanners. It might be accounted for by imaging reference nodules at only a few locations within the lung (e.g., at the center, near the spine, and near the upper periphery.)

\section{CONCLUSION}

This study demonstrates that even with state-of-the-art CT scanners, the CT numbers of simulated lung nodules are highly dependent upon the size of the nodule and the amount of air surrounding the nodule. Furthermore, for small nodules, the CT number is highly dependent upon the scan technique. In particular, one of the scanning protocols, that of the National Lung Screening Trial (NLST), utilizes $2.5 \mathrm{~mm}$ thick slices and $2 \mathrm{~mm}$ slice intervals, which were shown to yield very inaccurate and imprecise CT numbers for simulated spherical nodules of about $5 \mathrm{~mm}$ in diameter. Use of 
thinner slice and smaller slice interval CT images (e.g., $\leqslant 1.25 \mathrm{~mm}$ slice and slice interval) that can be reconstructed retrospectively from the original NLST protocol multidetector helical CT projections yield results that are more accurate and more reproducible. Until manufacturers develop CT scanners that are quantitative or have a quantitative reconstruction mode that incorporates sophisticated $\mathrm{X}$-ray beam hardening and $\mathrm{x}$-ray scatter corrections, reference phantom sets (anthropomorphic sections of various sizes with calibration nodules of known calcium compositions and sizes) similar to the phantom set employed in this study should be utilized to better characterize the calcium content of nodules via either manual or computerized analysis of images. Such phantoms can also be used to correct for variations in CT numbers between scanners of different makes and models and for differences in scanning protocols (e.g., $\mathrm{kVp}$ and reconstruction kernel). These corrections are important both for the initial characterization of the nodules and for long-term longitudinal studies in which the nodules are scanned in multiple exams using scanners that may not be identical or have identical response. Finally, corrected CT numbers and calcium contents derived with a reference phantom technique may prove to be valuable features in CAD analysis of lung nodules. The accuracies, universal applicabilities, and consistencies of the CAD schemes should be improved with the use of such methods.

\section{ACKNOWLEDGMENTS}

This work was supported in part by USPHS Grant No. CA93517. Its contents are solely the responsibility of the authors and do not necessarily represent the official views of the funding agency and no official endorsement of any equipment and product of any companies mentioned should be inferred.

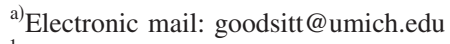

${ }^{1}$ W. R. Webb, "Radiological evaluation of the solitary pulmonary nodule," AJR, Am. J. Roentgenol. 154, 701-708 (1990).

${ }^{2}$ R. D. Tarver, R. W. Holden, and J. H. Ellis, "Experimental lung nodule model: CT numbers, nodule size and actual calcium content," J. Comput. Assist. Tomogr. 7, 402-406 (1983).

${ }^{3}$ E. A. Zerhouni et al., "A standard phantom for quantitative CT analysis of pulmonary nodules," Radiology 149, 767-773 (1983).

${ }^{4}$ E. A. Zerhouni et al., "CT of the pulmonary nodule: A cooperative study," Radiology 160, 319-327 (1986)

${ }^{5}$ S. S. Siegelman, E. A. Zerhouni, F. P. Leo, N. F. Khouri, and F. P. Stitik, "CT of the solitary pulmonary nodule," AJR, Am. J. Roentgenol. 135, 1-13 (1980).

${ }^{6}$ S. J. Swensen, G. F. Harms, R. L. Morin, and J. L. Myers, "CT evaluation of solitary pulmonary nodules: Value of $185-\mathrm{H}$ reference phantom," AJR, Am. J. Roentgenol. 156, 925-929 (1991).

${ }^{7}$ A. Khan, P. G. Herman, P. Vorwerk, P. Stevens, K. A. Rojas, and M. Garver, "Solitary pulmonary nodules: Comparison of classification with standard, thin-section and reference phantom CT," Radiology 179, 477481 (1991).

${ }^{8}$ M. Hasegawa, S. Sone, S. Takashima, F. Li, Z.-G. Yang, Y. Maruyama, and T. Watanabe, "Growth rate of small lung cancers detected on mass CT screening,” Br. J. Radiol. 73, 1252-1259 (2000).

${ }^{9}$ J. Gohagan et al., "Final results of the lung screening study, a randomized feasibility study of spiral CT versus chest x-ray screening for lung cancer," Lung Cancer 47, 9-15 (2005).

${ }^{10}$ National Bureau of Standards, "Physical aspects of irradiation," ICRU Report 10b. Handbook 85 (1964).

${ }^{11}$ W. A. Kalender, R. Rienmuller, W. Seissler, J. Behr, M. Welke, and H. Fichte, "Measurement of pulmonary parenchymal attenuation: Use of spirometric gating with quantitative CT," Radiology 175, 265-268 (1990).

${ }^{12}$ CRC Handbook of Chemistry and Physics, 56th ed. (CRC, Cleveland, OH, 1975), p. D-81.

${ }^{13}$ S. K. Shah, M. F. McNitt-Gray, S. R. Rogers, J. G. Goldin, R. D. Suh, J. W. Sayre, I. Petkovska, J. J. Kim, and D. R. Aberlee, "Computer aided characterization of the solitary pulmonary nodule using volumetric and contrast enhancement features," Acad. Radiol. 12, 1310-1319 (2005). 